Unidad de Medicina MaternoFetal, División de Obstetricia y Ginecología, Escuela de Medicina, Pontificia Universidad Católica de Chile. Santiago, Chile.

Conflictos de intereses: nada que declarar.

Recibido el 6 de agosto de 2013, aceptado el 16 de enero de

2014

Correspondencia a:

Dr. Enrique Donoso

Lira $85,5^{\circ}$ piso. Santiago, Chile. Teléfono: (56-2) 354-3034

edonoso@med.puc.c.

\section{La edad de la mujer como factor de riesgo de mortalidad materna, fetal, neonatal e infantil}

\author{
ENRIQUE DONOSO, JORGE A. CARVAJAL, \\ CLAUDIO VERA, JOSÉ A. POBLETE
}

\section{Women age as a risk factor for maternal, fetal, neonatal and infant mortality}

\begin{abstract}
Background: Adolescent pregnancy and advanced maternal age are associated with increased risk for maternal, perinatal and infant death. However, the maternal age with the lowest reproductive risk has not been established. Aim: To determine the range of maternal age with the lowest reproductive risk. Material and Methods: A population-based study (2005-2010) was performed analyzing raw data from vital statistics yearbooks of the National Institute of Statistics of Chile. The association of maternal, fetal, neonatal and infant mortality with maternal age was analyzed. The latter was stratified in quinquenniums, between ages 10 and 54 years. Maternal, fetal, neonatal and infant mortality rates were calculated for each quinquennium. The lowest rate was selected as a control group for risk analysis, which was estimated according to Odds Ratio with 95\% confidence intervals. Results: Women of 20-29, 25-34 and under 30 years, had the lowest rate of fetal, neonatal/infant and maternal death, respectively. Women aged 45-49 years had the higher rate of maternal, fetal, neonatal and infant mortality. The risk of fetal, neonatal and infant mortality doubled from 40-44 years onwards, and maternal mortality from the age of 30-34 years. Conclusions: Our results suggest that the maternal age range with the lesser general reproductive risk is between 20-29 years. This finding should be considered in future studies of reproductive risk and for an appropriate counseling about conception.
\end{abstract}

(Rev Med Chile 2014; 142: 168-174)

Key words: Maternal age; Perinatal mortality; Pregnancy in adolescence; Sex counseling.
L a edad es un antecedente biodemográfico que permite identificar factores de riesgo a lo largo del ciclo vital de las personas; en el caso de la mujer adquiere especial importancia el período llamado edad fértil. Este período caracteriza a la mujer por su capacidad de ovular y embarazarse. El embarazo, si bien es una situación fisiológica, expone a la mujer, al feto y recién nacido, a la probabilidad de enfermar o morir.

El embarazo antes de los 20 y después de los 35 años, se asocia a un mayor riesgo materno y perinatal. El embarazo en menores de 20 años o embarazo adolescente, además del mayor riesgo biológico que implica ${ }^{1}$, genera una situación de riesgo social para el recién nacido y la madre ${ }^{2}$, siendo un importante problema de salud pública en la mayoría de los países, especialmente para aquellos en desarrollo ${ }^{3}$. El embarazo en mujeres de 35 o más años se asocia a un aumento del riesgo de presentar una serie de patologías propias de la gestación y una mayor frecuencia de patologías maternas crónicas, que traen como consecuencia una mayor probabilidad de muerte materna y perinatal ${ }^{4-8}$. 
El embarazo en mujeres de 35 o más años ha sido definido como embarazo en edad materna avanzada (advanced maternal age) $)^{5}$, aunque otros utilizan este término a partir de los 40 años $^{6}$, y como embarazo en edad muy avanzada a partir de los 45 años (very advanced maternal age, extremely advanced maternal age $)^{7,8}$, para resaltar la condición de mayor riesgo materno-perinatal de esas edades maternas. En Chile, se ha reportado el mayor riesgo de morbi mortalidad materna y perinatal del embarazo en adolescentes ${ }^{9} \mathrm{y}$ en edad materna avanzada ${ }^{10-12}$, concordante con publicaciones internacionales ${ }^{1,4-8}$.

No hemos encontrado estudios que comparen el riesgo materno-perinatal, en grupos etarios estratificados en rangos de edad materna a lo largo de la edad fértil, que permitan identificar el rango etario de menor riesgo reproductivo. Es así que los diferentes estudios utilizan como grupo control o de empírico menor riesgo, a mujeres de 20-24 años ${ }^{1}, 20-29$ años $^{6}, 20-34$ años $^{13}, 30-34$ años $^{7}$ y menores de 35 años $^{14}$, dificultando la comparación entre las investigaciones.

El objetivo de este estudio es determinar el quinquenio de edad materna con menor riesgo para los indicadores de mortalidad fetal, neonatal, infantil y materna, para así establecer los subgrupos etarios de referencia para futuros estudios de riesgo reproductivo y un adecuado consejo preconcepcional.

\section{Material y Método}

Estudio de base poblacional para determinar la tasa de mortalidad materna, fetal, neonatal e infantil, según rango de edad materna, a lo largo de la edad fértil de la mujer, entre los años 2005 y 2010. Los datos crudos fueron obtenidos de los Anuarios de Estadísticas Vitales de los años en estudio y publicados por el Instituto Nacional de Estadísticas de Chile ${ }^{15}$. Se definió para este estudio como edad fértil el período comprendido entre los 10 y 54 años.

En abril de $2003^{16}$, Chile incorporó los conceptos de nacido vivo (NV) y muerte fetal de acuerdo a lo establecido en la $10^{\text {a }}$ Clasificación Internacional de Enfermedades y Problemas relacionados con la Salud (CIE 10) ${ }^{17}$, que los define como independientes de la duración de la gestación $y$, por tanto, del peso al nacer. Debido a lo anterior y previo a definir el período de estudio, analiza- mos la tendencia de la notificación de los NV y muertos con peso inferior a $500 \mathrm{~g}$; observamos una tendencia significativamente ascendente en la notificación de los $\mathrm{NV}(\mathrm{r}=0,803 ; \mathrm{p}=0,000)$ y muertos $(r=0,802 ; p=0,000)$ entre los años 2003 y 2010, y sin cambio significativo entre los años 2005 y 2010 de los NV $(r=0,500 ; p=0,312)$ y muertos $(r=0,387 ; p=0,449)$. Por este motivo, seleccionamos como período de estudio el comprendido entre los años 2005 y 2010.

Se calcularon las tasas de mortalidad fetal, neonatal ( $<28$ días de vida), infantil ( $<1$ año de vida) y materna, en relación al número de $\mathrm{NV}$ ocurridos e inscritos en el año de nacimiento. La edad materna fue estratificada en quinquenios entre los 10 y 54 años. Para cada quinquenio se calculó la tasa de mortalidad fetal, neonatal, infantil y materna. La menor mortalidad según quinquenio de edad materna se usó como grupo control o de referencia, para el análisis de riesgo contra cada uno de los otros quinquenios. El análisis de riesgo se estimó según Odds Ratio (OR) con intervalo de confianza de 95\% (IC 95\%). El riesgo también se estimó con razón de tasas sin cambiar la estimación de la asociación por la baja incidencia de los eventos. Debido a que prácticamente todos los estudios publicados sobre el tema, y los propios ${ }^{9,10,12}$, usan OR, decidimos la primera metodología estadística. La comparación de proporciones se efectuó según ji cuadrado con corrección de Yates. Se estableció como significativo un valor $\mathrm{p}<0,05$.

\section{Resultados}

En el período 2005-2010 hubo 1.452.053 NV, 12.381 muertes fetales, 7.870 muertes neonatales, 11.566 muertes infantiles y 269 muertes maternas. Las tasas promedio de mortalidad fetal, neonatal, infantil y materna fueron: $8,53 / 1000 \mathrm{NV}$, $5,42 / 1000 \mathrm{NV}, 7,97 / 1000 \mathrm{NV}$ y $18,53 / 100.000 \mathrm{NV}$, respectivamente.

La Tabla 1 muestra el número de NV según rango de edad materna. Dieciseis coma doce por ciento correspondió a mujeres menores de 20 años, $67,78 \%$ a mujeres entre $20-34$ años, y $16,10 \%$ a mujeres de 35 o más años.

La Figura 1 muestra las tasas de mortalidad fetal, neonatal, infantil y materna, según quinquenio de edad de la madre. Las mujeres de 25-29 años presentaron la menor tasa de mortalidad fetal $(7,15 / 1000 \mathrm{NV})$ y neonatal $(4,66 / 1000 \mathrm{NV})$; 
mientras que la menor tasa de mortalidad infantil $(6,74 / 1000 \mathrm{NV})$ y materna $(8,76 / 100.000 \mathrm{NV})$ correspondió a las mujeres de 30-34 años y las adolescentes de 15-19 años, respectivamente. Las mujeres de 45-49 años presentaron las mayores tasas de mortalidad fetal $(28,6 / 1000 \mathrm{NV})$, neonatal $(12,75 / 1.000 \mathrm{NV})$, infantil $(20,3 / 1000 \mathrm{NV})$ y materna $(159,3 / 100.000 \mathrm{NV})$.

Tabla 1. Frecuencia de nacidos vivos según quinquenios de edad materna. Chile, 2005-2010

\begin{tabular}{|ccc|}
\hline $\begin{array}{c}\text { Edad materna } \\
\text { (años) }\end{array}$ & $\begin{array}{c}\text { Nacidos } \\
\text { vivos }\end{array}$ & $\begin{array}{c}\text { Frecuencia } \\
\text { (\%) }\end{array}$ \\
\hline $10-14$ & 5.905 & 0,41 \\
$15-19$ & 228.190 & 15,71 \\
\hline $20-24$ & 342.678 & 23,60 \\
$25-29$ & 340.937 & 23,48 \\
\hline $30-34$ & 300.579 & 20,70 \\
$35-39$ & 180.924 & 12,46 \\
$40-44$ & 50.296 & 3,46 \\
$45-49$ & 2.510 & 0,17 \\
\hline $50-54$ & 34 & 0,002 \\
\hline Total & 1.452 .053 & 100,0 \\
\hline
\end{tabular}

La Tabla 2 presenta el análisis de riesgo de mortalidad entre el grupo control (menor tasa de mortalidad) y el resto de los rangos etarios. Muestra un riesgo mayor de mortalidad en los grupos extremos de edad reproductiva (10-14 años y 45-49 años), excepto la mortalidad materna en adolescentes de 10-14 años que si bien es alta $(33,87 / 100.000 \mathrm{NV})$ no tiene diferencia significativa respecto al grupo control. Se excluye del análisis a las mujeres de 50 o más años por bajo número de casos (NV: 34 ; muertes fetales: 12; muertes neonatales: 9; muertes infantiles: 12 ; muertes maternas: 0 ).

\section{Mortalidad fetal}

En relación al grupo control (25-29 años) no hubo diferencia significativa con el grupo 20-24 años. En todos los otros rangos de edad materna el riesgo fue significativamente mayor, duplicándose en las de 40-44 años y cuadruplicándose en las de 45-49 años (Tabla 2).

\section{Mortalidad neonatal}

En relación al grupo control (25-29 años) no hubo diferencia significativa con el grupo 30-34 años. En todos los otros rangos de edad materna el riesgo fue significativamente mayor, duplicándose
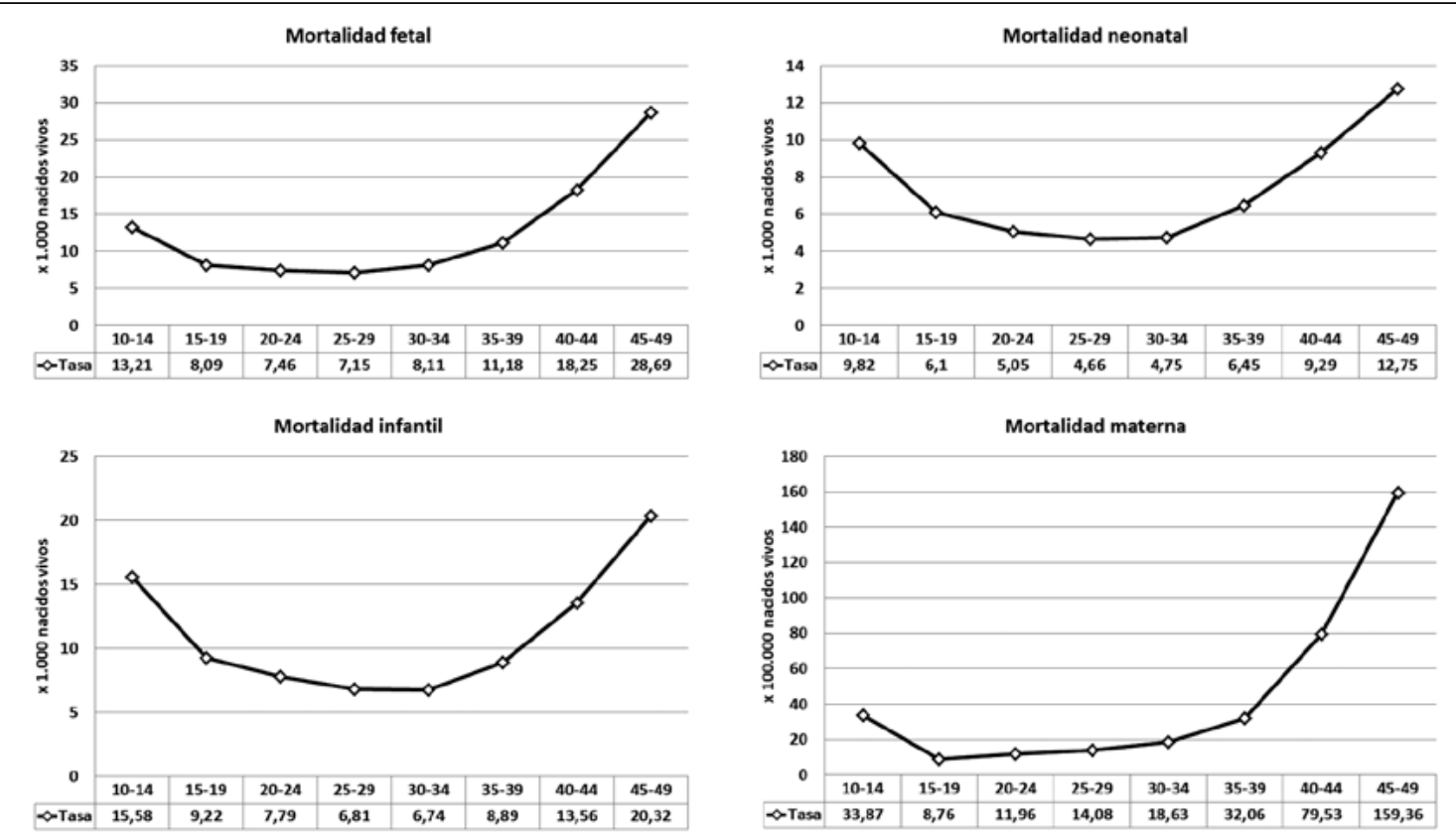

Figura 1. Tendencia de la mortalidad fetal, neonatal, infantil y materna según rangos de edad materna. Chile, 2005-2010. 
Edad materna y riesgo reproductivo - E. Donoso et al

Tabla 2. Análisis de riesgo de mortalidad fetal, neonatal, infantil y materna, según rangos de edad materna. Chile, 2005-2010

\begin{tabular}{|ccccc|}
\hline $\begin{array}{c}\text { Edad materna } \\
\text { (años) }\end{array}$ & $\begin{array}{c}\text { Mortalidad fetal } \\
\text { OR (IC95\%) }\end{array}$ & $\begin{array}{c}\text { Mortalidad neonatal } \\
\text { OR (IC95\%) }\end{array}$ & $\begin{array}{c}\text { Mortalidad infantil } \\
\text { OR (IC95\%) }\end{array}$ & $\begin{array}{c}\text { Mortalidad materna } \\
\text { OR (IC95\%) }\end{array}$ \\
\hline $10-14$ & $1,85(1,46-2,33)$ & $2,12(1,61-2,78)$ & $2,33(1,88-2,89)$ & $3,86(0,90-16,53)^{*}$ \\
\hline $15-19$ & $1,13(1,07-1,20)$ & $1,31(1,22-1,41)$ & $1,37(1,29-1,46)$ & 1 \\
\hline $20-24$ & $1,04(0,99-1,11)^{*}$ & $1,08(1,01-1,16)$ & $1,16(1,09-1,23)$ & $1,37(0,78-2,41)^{*}$ \\
\hline $25-29$ & 1 & 1 & $1,01(0,95-1,07)^{*}$ & $1,61(0,93-2,80)^{*}$ \\
\hline $30-34$ & $1,13(1,07-1,20)$ & $1,02(0,95-1,10)^{*}$ & 1 & $2,13(1,25-3,66)$ \\
\hline $35-39$ & $1,56(1,47-1,66)$ & $1,39(1,29-1,50)$ & $1,32(1,24-1,41)$ & $3,66(2,15-6,28)$ \\
\hline $40-44$ & $2,55(2,36-2,76)$ & $2,00(1,80-2,22)$ & $2,03(1,86-2,21)$ & $9,08(5,16-16,08)$ \\
\hline $45-49$ & $4,01(3,14-5,12)$ & $2,76(1,91-3,97)$ & $3,06(2,28-4,08)$ & $18,21(5,27-56,30)$ \\
\hline
\end{tabular}

*No significativo.

en las menores de 15 años y en las de 40-44 años, y casi triplicándose en las de 45-49 años (Tabla 2).

\section{Mortalidad infantil}

No hubo diferencia significativa entre el grupo 25-29 años y el grupo control (30-34 años). En todos los otros rangos de edad materna el riesgo fue significativamente mayor, duplicándose en las menores de 15 años y en las de 40-44 años, y triplicándose en las de 45-49 años (Tabla 2).

\section{Mortalidad materna}

No hubo diferencia significativa en los grupos 10-14, 20-24 y 25-29 años comparados con el grupo control (30-34 años). En los otros rangos de edad materna el riesgo fue significativamente mayor, duplicándose en las de 30-34 años y fue 18 veces mayor en las de 45-49 años (Tabla 2).

\section{Discusión}

Nuestros resultados indican que el rango de edad materna con menor riesgo de mortalidad fetal estuvo entre los 20 y 29 años, neonatal e infantil entre los 25 y 34 años, y materna en menores de 30 años. Se establece que el grupo de mujeres entre 20 y 29 años es el rango etario que concentra el menor riesgo de mortalidad materna, fetal, neonatal e infantil, antecedente importante para futuros estudios de riesgo reproductivo y un apropiado consejo preconcepcional.

Los rangos de edad materna fueron estratifi- cados en quinquenios, pues así es la información entregada por el INE. Reconocemos que, al ser la edad materna una variable continua, es posible que dentro de un quinquenio, el riesgo pueda ser mayor a mayor edad de la mujer. Sin embargo, en forma pragmática utilizamos los mismos quinquenios reportados por el INE.

\section{Embarazo en mujeres menores de 20 años}

El grupo de adolescentes de 10-14 años fue uno de los rangos de mayor mortalidad materna, fetal, neonatal e infantil, originando las clásicas formas en "J" o en "U" de las curvas de mortalidad (Figura 1). La tasa de mortalidad materna en adolescentes de 10-14 años, pese a ser una de las más altas $(33,86 / 100.000 \mathrm{NV})$ no fue significativamente mayor que la del grupo control (OR 3,86; IC95\% 0,90-16,53), estimación limitada por el bajo número de casos en un estrato etario con baja frecuencia del evento ( 2 muertes maternas). Sin embargo, las adolescentes de 15-19 años son el grupo de menor mortalidad materna $(8,76 / 100.000 \mathrm{NV})$, muy inferior al promedio del período $(18,57 / 100.000$ NV). Postulamos que esta baja tasa de mortalidad materna para adolescentes de 15-19 años se debe a que las adolescentes chilenas no recurren habitualmente al aborto inducido para controlar su fecundidad ${ }^{18}$.

En general, nuestros resultados son concordantes con lo reportado por la literatura internacional. Estudios poblacionales efectuados en Estados Unidos de Norteamérica notifican un mayor riesgo de bajo peso al nacer, parto prematuro y restricción 
del crecimiento fetal ${ }^{1,19,20}$ en adolescentes de 13-17 años $^{1}, 10-19$ años $^{19}$ y menores de 15 años ${ }^{20,21}$, lo que explicaría la mayor mortalidad neonatal ${ }^{19,20}$, infantil $^{19,20} \mathrm{y}_{\text {fetal }}{ }^{13,21,22}$. Un estudio poblacional sueco ${ }^{23}$ reportó un mayor riesgo de mortalidad neonatal y post neonatal en adolescentes de 13-15 años y 16-17 años, mientras que en el grupo de 1819 años sólo hubo un mayor riesgo en mortalidad post neonatal. En Brasil ${ }^{24}$ se reportó un mayor riesgo de mortalidad post neonatal e infantil en adolescentes de 12-19 años, sin diferencias en mortalidad fetal, neonatal y perinatal, lo que fue atribuido a factores sociales y ambientales adversos. Un estudio británico ${ }^{25}$ reportó en menores de 18 años un significativo mayor riesgo de parto prematuro menor de 32 semanas, y sin diferencias en mortalidad fetal y recién nacidos pequeños para la edad gestacional. Una revisión sistemática publicada en $2001^{26}$, sugirió que el mayor riesgo perinatal de las adolescentes de 16-19 años estaba marcado por factores sociales, económicos y ambientales, mientras que en las menores de 16 años hubo un mayor riesgo de prematurez, bajo peso al nacer y mortalidad neonatal, independiente de los factores socioeconómicos y ambientales.

Anteriormente, reportamos en un estudio poblacional (1990-1999) el riesgo perinatal de las adolescentes chilenas comparadas con adultas de 20-34 años 9 . Las adolescentes de 10-14 años y de 15-19 años presentaron una mortalidad neonatal e infantil significativamente mayor que las mujeres de 20-34 años. En las adolescentes de 10-14 años el riesgo de mortalidad fetal tardía y de mortalidad materna no fue significativamente mayor que el del grupo control, mientras que en adolescentes de 15-19 años el riesgo de mortalidad materna y fetal tardía fue significativamente menor que el de las mujeres de 20-34 años. Las diferencias de los resultados de ese estudio con el actual pueden explicarse por corresponder a diferentes períodos (1990-1999 y 2005-2010): en el primero se estudió la mortalidad fetal tardía ( $\geq 28$ semanas) y en el segundo la mortalidad fetal independiente de la edad gestacional.

El embarazo adolescente es un grave problema de salud pública en países desarrollados y en desarrollo, especialmente concentrado en los grupos sociales más vulnerables, que tienen bajo acceso a programas de educación sexual y anticoncepción ${ }^{27,28}$. En esta investigación observamos que en Chile, el embarazo adolescente constituye 16,1\%
(234.095/1.452.053) de los nacidos vivos, $8,2 \%$ (22/269) de las muertes maternas y una mortalidad materna en menores de 20 años de 9,4/100.000 $\mathrm{NV}(22 / 234.095 \mathrm{NV})$, cifra inferior a las promedio reportadas para Latinoamérica ${ }^{3}$. Para lograr una reducción de estas cifras se requiere de efectivas políticas públicas educacionales y sanitarias que permitan el retraso del inicio de la vida coital y la prevención del embarazo no planificado, siendo este último la principal causa de deserción escolar y de perpetuación de la pobreza en adolescentes chilenas $^{2,29,30}$. El control de la fecundidad en adolescentes, permitirá disminuir la mortalidad materna, fetal, neonatal e infantil, tal como es sugerido por WHO y $\mathrm{UNFPA}^{31}$.

\section{Embarazo en mujeres de 20-34 años}

Dentro de este rango etario las diferentes investigaciones seleccionaron empíricamente los grupos control para los análisis de riesgo ${ }^{1,6,7,9,10,12,13}$, sin existir una demostración de cuál es el mejor grupo a utilizar. Ahí radica la importancia de esta investigación, que identifica los grupos etarios de menor riesgo de mortalidad materna, fetal, neonatal e infantil, permitiendo elegir el mejor grupo control para los análisis de riesgo. En nuestro estudio observamos que el grupo de mujeres de 30-34 años, presenta un mayor riesgo de mortalidad fetal y materna que su respectivo grupo control (Tabla 2), sin embargo, frecuentemente ese grupo etario es incluido como parte del grupo control para los análisis de riesgo $0^{1,7,9,10,12,13,23}$. Nuestros resultados muestran que para futuros estudios de riesgo reproductivo el grupo de mujeres entre 20-29 años concentra el menor riesgo de mortalidad materna, fetal, neonatal, e infantil y recomendamos su uso como grupo de referencia.

\section{Embarazo en mujeres de 35 o más años}

Demostramos claramente una mayor mortalidad fetal, neonatal, infantil y materna en los grupos de mujeres de 35 o más años. Anteriormente habíamos comunicado el alto riesgo de mortalidad materna, perinatal e infantil en mujeres de 40 o más años ${ }^{10}$, y perinatal e infantil en las de 50 o más años $^{12}$, comparadas con mujeres de 20-34 años. Asimismo, reportamos la natalidad significativamente ascendente en las mujeres mayores de 40 años $^{32}$, que trajo como consecuencia un cambio del perfil epidemiológico de la población obstétrica chilena ${ }^{33} \mathrm{y}$ un estancamiento de los indicadores 
de mortalidad materna y perinatal. En el actual estudio no analizamos el riesgo en mujeres de 50 o más años, por un insuficiente número de casos, sin embargo, una serie reportada por nosotros sobre un total de 213 nacidos vivos, demostró en ellas el mayor riesgo de mortalidad fetal, neonatal e infantil ${ }^{12}$.

Nuestros resultados concuerdan con estudios internacionales, pese a la diferencia etaria de los grupos controles, al confirmar el mayor riesgo reproductivo en mujeres de 35, 40 y 45 o más años ${ }^{5-8,13,14,34,35}$. Son escasos los estudios en mujeres de 50 o más años con gestaciones espontáneas ${ }^{12,36}$, mientras que otros corresponden a estudios descriptivos de embarazos por ovo donación en mujeres seleccionadas ${ }^{37,38}$.

Debemos ser cautos en el uso clínico del conocimiento del mayor riesgo materno-perinatal en mujeres en edad materna avanzada, al momento del consejo reproductivo preconcepcional, para no generar ansiedad y miedo en su decisión ${ }^{39}$. La postergación de la maternidad a edades avanzadas es un fenómeno demográfico característico de los países desarrollados y en los cercanos al desarrollo, por lo que debemos respetar las decisiones de las mujeres debidamente informadas con un adecuado consejo preconcepcional. Consideramos pertinente sugerir la generación de efectivas políticas públicas que incentiven el embarazo en edades maternas de menor riesgo reproductivo y social.

\section{Conclusión}

Nuestra investigación determinó que el rango de edad materna de menor riesgo de mortalidad fetal fue entre los 20-29 años, neonatal e infantil entre los 25-34 años, y materna en menores de 30 años, siendo el grupo de mujeres entre 20-29 años las que concentran el menor riesgo de mortalidad materna, fetal, neonatal e infantil. Sugerimos que ese rango etario sea usado para comparaciones en estudios de riesgo reproductivo, como también para un adecuado consejo preconcepcional. Las mujeres de 40 o más años presentaron las tasas más altas de mortalidad materna, fetal, neonatal e infantil.

\section{Referencias}

1. Fraser AM, Brockert JE, Ward RH. Association of young maternal age with adverse reproductive outcomes. $\mathrm{N}$
Engl J Med 1995; 332 (17): 1113-7.

2. Molina R, Molina T, González E. [Teenage fecundity rates in Chile: a serious public health problem] Rev Med Chile 2007; 135 (1): 79-86. [Article in Spanish].

3. Conde-Agudelo A, Belizán JM, Lammers Q. Maternalperinatal morbidity and mortality associated with adolescent pregnancy in Latin America: Cross-sectional study. Am J Obstet Gynecol 2005; 192 (2): 342-9.

4. Cleary-Goldman , Malone FD, Vidaver , Ball RH, Nyberg DA, Comstock CH et al; FASTER Consortium. Impact of maternal age on obstetric outcome. Obstet Gynecol 2005; 105 (5 Pt 1): 983-90.

5. Huang I, Sauve R, Birkett N, Fergusson D, van Walra ven $\mathrm{G}$ Maternal age and risk of stillbirth: a systematic review. CMA] 2008; 178 (2): 165-72.

6. Kenny LC, Lavender T, McNamee R, O’Neill SM, Mills T, Khashan AS. Advanced maternal age and adverse pregnancy outcome: evidence from a large contemporary cohort PLoS One 2013; 8 (2): e56583.

7. Carolan MO, Davey MA, Biro M, Kealy M Very advanced maternal age and morbidity in Victoria, Australia: a population based study. BMC Pregnancy Childbirth 2013; 13 (1): 80.

8. Yogev Y, Melamed N, Bardin R, Tenenbaum-Gavish K, Ben-Shitrit G, Ben-Haroush A. Pregnancy outcome at extremely advanced maternal age. Am J Obstet Gyneco] 2010; 203 (6): 558. e1-7.

9. Donoso Siña E, Becker Valdivieso J, Villarroel del Pino L. [Birth rates and reproductive risk in adolescents in Chile, 1990-1999], Rev Panam Salud Pública 2003; 14 (1): 3-8. [Article in Spanish].

10. Donoso E, Villarroel L. Reproductive risk of women over 40 years old]. Rev Med Chile 2003; 131 (1): 55-9. [Article in Spanish].

11. Chamy V, Cardemil F, Betancour P, Ríos M, Leigthon L. Riesgo obstétrico y perinatal en embarazadas mayores de 35 años. Rev Chil Obstet Ginecol 2009; 74 (6): 331-8.

12. Donoso E, Carvajal JA. Maternal, perinatal and infant outcome of spontaneous pregnancy in the sixth decade of life Maturitas 2008; 59 (4): 381-6.

13. Bateman BT, Simpson LL. Higher rate of stillbirth at the extremes of reproductive age: a large nationwide sample of deliveries in the United States. Am J Obstet Gynecol 2006; 194 (3): 840-5.

14. Reddy UM, Ko CW, Willinger M Maternal age and the risk of stillbirth throughout pregnancy in the United States. Am J Obstet Gynecol 2006; 195 (3): 764-70.

15. Instituto Nacional de Estadísticas, Servicio de Registro Civil e Identificación, Departamento de Estadísticas e Información de Salud. Estadísticas Vitales. Informes anuales 2005-2010. Disponibles en: http://www.ine.cl 
canales/chile_estadistico/demografia_y_vitales/estadisticas_vitales/estadisticas_vitales.php. [Consultado el 02 de noviembre de 2012].

16. Gobierno de Chile, Ministerio de Salud, Departamento de Estadísticas e Información en Salud. Ord. 17 A N ${ }^{\circ}$ 1873. Disponible en: http://deis.minsal.cl/deis/codigo/ neuw/Norma_fetales/1873_17A03_09_04_03.pdf. [Consultado el 23 de febrero de 2013.

17. Organización Mundial de la Salud, Organización Panamericana de la Salud. Clasificación Estadística Internacional de Enfermedades y Problemas Relacionados con la Salud. Décima Revisión. Volumen 1. Publicación Científica No 554. Washington DC, 1995. Disponible en: http://apps.who.int/classifications/apps/icd/icd10onlinet. [Consultado el 3 de febrero de 2011].

18. Silva S. Aborto. En: Pérez Sánchez A, Donoso Siña E, Editores, Obstetricia (4a Edición). Santiago, Chile: Editorial Mediterráneo Ltda.; 2011. p 530-61.

19. Chen XK, Wen SW, Fleming N, Demissie K, Rhoads GG, Walker M. Teenage pregnancy and adverse birth outcomes: a large population based retrospective cohort study. Int J Epidemiol 2007; 36 (2): 368-73.

20. Malabarey OT, Balayla J, Klam SL, Shrim A, Abenhaim HA. Pregnancies in young adolescent mothers: a population-based study on 37 million births. J Pediatr Adolesc Gynecol 2012; 25 (2): 98-102.

21. Menacker F, Martin JA, MacDorman MF, Ventura SJ. Births to 10-14 year-old mothers, 1990-2002: trends and health outcomes. Natl Vital Stat Rep 2004; 53 (7): 1-18.

22. Salihu HM, Sharma PP, Ekundayo O, Kristensen S, Badewa AP, Kirby RS, et al. Childhood pregnancy (10-14 years old) and risk of stillbirth in singletons and twins. [ ] Pediatr 2006; 148 (4): 522-6.

23. Olausson PO, Cnattingius \$, Haglund B. Teenage pregnancies and risk of late fetal death and infant mortality. Br J Obstet Gynaecol 1999; 106 (2): 116-21.

24. Restrepo-Méndez MQ Barros A, Santos IS, Menezes AM, Matijasevich A, Barros FO, et al. Childbearing during adolescence and offspring mortality: findings from three population-based cohorts in southern Brazil. BMQ Public Health 2011; 11: 781.

25. Jolly MQ Sebire N, Harris ], Robinson S, Regan I. Obstetric risks of pregnancy in women less than 18 years old. Obstet Gyneco 2000; 96 (6): 962-6.

26. Cunnington A]. What's so bad about teenage pregnancy? J Fam Plann Reprod Health Care 2001; 27 (1): 36-41.

27. World Health Organization, Department of Child and Adolescent Health and Development, Department of Reproductive Health and Research. Adolescent Preg- nancy. Issues in Adolescent Health and Development. Geneva, 2004. Disponible en: http://whqlibdoc.who.int/ publications/2004/9241591455_eng.pdt. [Consultado el 22 de octubre de 2012].

28. Molina Cartes R, González Araya $\mathrm{E}$ Teenage pregnancy. Endocr Dev 2012; 22: 302-31.

29. Molina M, Ferrada O, Pérez R, Cid I Casanueva V, García A [The relationship between teenage pregnancy and school desertion]. Rev Med Chile 2004; 132 (1): 65-70. [Article in Spanish].

30. Gobierno de Chile, Ministerio de Salud. Situación actual del embarazo adolescente en Chile. Mayo de 2013. Disponible en: http://www.minsal.cl/portal/url/item/ c908a2010f2e7dafe040010164010db3.pdf. [Consultado el 28 de mayo de 2013].

31. World Health Organization (WHO), United Nations Fund for Population Activities (UNFPA). Pregnant Adolescents: delivering on global promises of hope. Disponible en: http://whqlibdoc.who.int/publications/ 2006/9241593784_eng.pdt [Consultado: 1 de diciembre de 2013].

32. Donoso E, Carvajal J, Domínguez MA. [Fecundity reduction and aging in fertile women population in the period 1990-2004 in Chile]. Rev Med Chile 2009; 137 (6): 766-73. [Article in Spanish].

33. Donoso E, Carvajal J. [The change in the epidemiological profile of maternal mortality in Chile will hinder the fulfillment of the Millennium $5^{\text {th }}$ goal]. Rev Med Chile 2012; 140 (10): 1253-62. [Article in Spanish].

34. Sutan R, Campbell D, Prescott G, Smith WQ The risk factors for an explained antepartum stillbirths in Scotland, 1994 to 2003. Derinato 2010; 30 (5): 311-8.

35. Schoen $\mathrm{Q}$ Rosen $\mathrm{T}$. Maternal and perinatal risks for women over 44-a review. Maturitas 2009; 64 (2): 109-13.

36. Narayan H, Buckett W, McDougall W, Cullimore ]. Pregnancy after fifty: profile and pregnancy outcome in a series of elderly multigravidae. Eur J Obstet Gynecol Reprod Biol 1992; 47 (1): 47-51.

37. Sauer MV, Paulson RJ, Lobo RA. Pregnancy in women 50 or more years of age: outcomes of 22 consecutively established pregnancies from oocyte donation. Fertil Steril 1995; 64 (1): 111-5.

38. Paulson RJ, Boostanfar R, Saadat P, Mor E, Tourgeman DE, Slater CC, et al. Pregnancy in the sixth decade of iife: obstetric outcomes in women of advanced reproductive age. JAMA 2002; 288 (18): 2320-3.

39. Benzies KM Advanced maternal age: are decisions about the timing of child-bearing a failure to understand the risks? CMA] 2008; 178 (2): 183-4. 